\title{
Research on CCO of Rat Myocardial by Intermittent Hypoxia Training
}

\author{
Fangtao Liu, Qiaozhen Yan \\ Wenshan County, Yunnan Province 65700, China \\ Liufangtao688@126.com
}

Keywords: Intermittent, Low oxygen, CCO

\begin{abstract}
Cytochrome oxidase is the key enzymes of body's aerobic metabolism, by measuring the activity of CCO, To understand the change of the aerobic capacity in rats. The results showed that: by intermittent hypoxia training, the rat myocardial cytochrome oxidase have adaptability to changes, shows that intermittent hypoxia training can effectively improve the rat myocardial aerobic capacity.
\end{abstract}

\section{Introduction}

Altitude training, which put athletes to appropriate altitude to have regular specialized sports training. Its theoretical basis is that the human body in the plateau, low pressure, low oxygen, by plateau hypoxia and exercise double stimulation, make the athletes produces strong stress reaction, to mobilize the body's function of potential, and resulting in a series of anti hypoxia physiological reaction to improve exercise capacity. But altitude training is a "double-edged sword", in raising the ability of human movement also brought some shortage at the same time, the altitude training of oxygen load and training load hard to co-exist at the same time, in order to solve this contradiction, simulation altitude training arises at the historic moment, let the athletes in the different time have sports training load and lack of oxygen load respectively, namely after oxygen under often trained to accept below quiet condition of oxygen load simulated plateau environment. Make originally in the altitude training mutual restriction factors in synergy, to maximize mobilize the body's ability, promote the improvement of aerobic endurance, among them with intermittent hypoxia training and high low applied most widely practiced. Domestic and foreign studies of skeletal muscle metabolic enzymes reported that by intermittent hypoxia training, the rat skeletal muscle, myocardial oxygen metabolism enzyme activity (MDH [1], SDH and CCO [2]) increased significantly, movement time extended. Increasing load of intermittent hypoxia exercise rats myocardial ATPase activity increased obviously, and prolong swimming time [3].

Shows that reasonable intermittent hypoxia training can make the muscle cells are appropriate stimulation, make muscle cell adaptive changes in the structure and function, muscle cells oxidase activity increases, organizations use oxygen ability enhancement, is advantageous to the myocardial and skeletal muscle aerobic metabolism, ensure the contraction of the muscle tissue energy supply, improve the working efficiency of the muscles and exercise lasting ability. In this paper, we study the key enzyme of aerobic metabolism enzyme CCO, explore the change of CCO in the intermittent hypoxia training, so as to explore the intermittent hypoxia training influence on aerobic capacity, provide the basis for sports training.

\section{The Research Content and Method}

The object of study. 72 SD male rats, 8 weeks, according to the experimental animal standard feeding, adapt to the training 1 week were randomly divided into 4 groups, formal training for three weeks, total of 4 weeks.

The Experimental Method. The experimental group Control group (C), Sports group (S), intermittent hypoxic control group (I), intermittent hypoxic training group (IS) and 18 in each group. 


\section{Training Arrangement.}

(1)The control group (C) : freefood and free drinking water without any training activities.

(2)Sports group(S) : rats in aerobic endurance training each day.

(3)(3)Intermittent hypoxic control group (I):

(4)Hypoxia group in low oxygen chamber IHT, three times a week, 1 hour each day (a combination of low oxygen for 5 minutes, 5 minutes intermittent, a total of six combination). Oxygen concentration was $14 \%$, the first week after week, weekly decline $1 \%$.

(5)Intermittent hypoxic training group (IS):Hypoxia group in low oxygen chamber IHT, three times a week, 1 hour each day (a combination of low oxygen for 5 minutes, 5 minutes intermittent, a total of six combination). Oxygen concentration was $14 \%$, the first week after week, weekly decline 1\%,for aerobic endurance training immediately after hypoxic training every day.

Movement Patterns: sports group in domestic rats electric treadmill training, the first week 15 $\mathrm{m} / \mathrm{min}, 30$ min every day, make animals familiar treadmill exercise, after a week, increasing load per week, Increased the speed of $5 \mathrm{~m} / \mathrm{min}, 10 \mathrm{~min}$; The last week of speed up to $30 \mathrm{~m} / \mathrm{min}$, the time for $60 \mathrm{~min}$.

The Experimental Materials. After the training, with $0.4 \%$ pentobarbital $1 \mathrm{ml} / 100 \mathrm{~g}$ weight, to anesthesia in rats, quickly remove the calf muscles and heart, the heart weighing, and then separate left and right ventricle, left ventricular wall thickness is measured with vernier caliper, gastrocnemius muscle and myocardial put into $-70{ }^{\circ} \mathrm{C}$ refrigerator.

Test Steps. Accurate weigh myocardial, posterior calf muscles of each $200 \mathrm{mg}$, respectively in small beaker $5 \mathrm{ml}$; With pipettor move $1 \mathrm{ml} 9 \%$ saline water which in advance in the refrigerator (4 ${ }^{\circ} \mathrm{C}$ ) precooling, scissors cut up as soon as possible organization block (small beaker into ice water).Pour into a glass with a cut up tissue homogenate in the tube, then take $0.8 \mathrm{ml}$ cold saline flushing residual tissue fragments in the beaker, homogenate together, make organization fully homogenate, made from $10 \%$ homogenate. And then divided into two parts, part in refrigerated centrifuge $3000 \mathrm{r} / \mathrm{min}$ for $10 \mathrm{~min}$, take supernatant, according to the conventional method for determination of the cytochrome oxidase centrifugal after determination of the activity of cytochrome oxidase.

Data Processing. All the data using the mean and standard deviation(, $\mathrm{X} \pm \mathrm{SD})$, statistical processing with single factor analysis of variance method, all data are calculated on a computer using statistical software SPSS and, significant difference of $\mathrm{P}<0.05$.

\section{Results}

The influence on on rat myocardial cytochrome oxidase by IHT From table 1 in the myocardial tissue of cytochrome oxidase changes: I group and $\mathrm{C}$ group have a very significant difference, $(\mathrm{P}<$ 0.01), IS group compare to $\mathrm{C}$ group, $\mathrm{S}$ group, I group have a very significant difference, $(\mathrm{P}<0.01)$.

Table 1:the influence on on rat myocardial cytochrome oxidase by $\operatorname{IHT}\left(\mathbf{K m i n}^{\mathbf{- 1}} \mathbf{m} \mathbf{m g}\right)$

\begin{tabular}{l|ll|l}
\hline Group & Quiet & After Sport & Recovery \\
\hline & & & \\
C & $4.40 \pm 0.44$ & $5.74 \pm 1.13$ & $4.85 \pm 0.53$ \\
S & $5.22 \pm 0.32^{\# \#}$ & $5.90 \pm 0.26$ & $5.23 \pm 0.25$ \\
I & $5.18 \pm 0.49^{\# \#}$ & $6.07 \pm 0.49$ & $5.67 \pm 1.39$ \\
IS & $6.18 \pm 0.26^{\# @ @ @ \Delta \Delta \text { 动 }}$ & $6.23 \pm 0.41$ & $5.70 \pm 1.03$ \\
\hline
\end{tabular}

\section{Analysis and Discussion}

$\mathrm{CCO}$ as the key enzyme in the respiratory chain of inner mitochondrial membrane and oxidative phosphorylation system, as a symbol of oxidation respiratory chain in immunohistochemical reaction ,pass electrons to molecular oxygen finally, is a carrier, at the end of the electron transport chain from earlier in the respiratory chain, electronic, through a series of process of the final state 
by the CCO to molecular oxygen (i.e., with automatic oxidation), the resulting water, release energy supply the body need [4].Electron transfer is the premise and foundation of ATP generation, and respiratory chain only generate ATP can promote electron transfer, both complement each other, as the key electron carrier, its content and activity of a direct impact on the respiratory chain efficiency of electron transfer process, directly affects the body's energy production and the level of aerobic metabolism. Mitochondria are cell respiration and the production of ATP, including NADH dehydrogenase, CCO and so on, electronic through these enzymes in the respiratory chain, eventually passed on from CCO to molecular oxygen (components of electron transport chain by REDOX reaction will provide the $\mathrm{NADH}$, in turn, pass down the final electronic accepted by oxygen molecules, reduction of water, at the same time release energy for the body need) [5].

In these enzymes cytochrome oxidase (CCO) is the enzyme, it is in cytochrome system, at the end of the last carrier of electron transport chain, including cytochrome a and a3, which pass the electron by cytochrome system to molecular oxygen (i.e., with automatic oxidation), lectron transfer and the formation of ATP in the normal tissue cells, which complement each other, ATP generated must be on the premise of electron transfer and the respiratory chain only generate ATP can promote electron transfer. Aerobic cells, sugar, fat and amino acid decomposition through their respective decomposition approach, but in the end all over a period of common oxidation [6].

In the process, sugar, fat and amino acid oxidation take off hydrogen to form a reduced coenzyme (NADH and FADH2), along the electron transport chain in the form of electronic and $\mathrm{H}$ + ions finally passed to the oxygen to form water, which release free energy that is used to make the ADP and Pi form ATP [7].Therefore CCO level determines the maximum working can be achieved when the body movement intensity and duration, it directly decided the endurance sports scores, this is the most important in sport, and the whole sports training goal [8]. Remarkable increase of the activity of CCO and endurance running training 4 weeks means that the ability of muscle cell oxidative phosphorylation, enhanced ability to increase in rat skeletal muscle fiber ATP storage, movement required for rats, HenriKssenet al. (1977) study proves that endurance training make human body muscle fibers CCO activity increased[9-10].

This experiment the results shows that: quiet condition, $\mathrm{C}$ group and the other three groups, $\mathrm{S}$ group and I group, I group and IS group, has a very significant difference $(\mathrm{P}<0.01)$, IS group myocardial CCO activity increased by $40 \%$. That movement of oxygen with pure oxygen for myocardial no significant difference, the effect of hypoxia stimulation of the environment in the motion of a form that the body cannot more extraneous stimulus and its become a source of stress, so it can't become an organism new stress reaction conditions. Immediately after exercise and exercise after three hours, there was no significant difference.

\section{Conclusion}

In this experiment, the intermittent hypoxia training can effectively improve the activity of rat myocardial CCO, complex movement group with low oxygen and training are obviously.

\section{References}

[1]S.C.Li,Y.Tian:Simulation Altitude Training's Impact on Metabolism of Skeletal Muscle in Mice,Vol.21(1994)No 2,p.15-18

[2]M.Y.Wang,Z.P.Lei:The Reserach of Effects of Aerobic Exercise Capacity in Mice by Intermittent Hypoxia training.2000,p:205-206

[3]Q.Li: The Reserach on Exercise Capacity by Different Ways of Intermittent Hypoxia.2000,p:207-208

[4]Q.B.Kong:Swimming Endurance Training for Different Weeks Myocardial Metabolic Enzymes in Mice.2007 
[5]Y.G.Zeng,J.H.He and X.G.Niu:Exercise Training on Zinc Copper Metabolism in Rats and Serum ALP, LDH Activity,Vol.26(2003)No.4,p:7-9

[6]Y.K.Zhang,Y.Jiao and W.Q.Feng:The Influence on Brain,Liver,Muscle Tissue Free Radical Metabolism and Serum CK,LDH in Mice by Exhaustion Swimming,Vol.6(1995)No.2,p:69-73

[7]DavidsonsR,BurnettM,Hoffman-GoetzL.Training effeetsi nmice after long-term Voluntary exercise.MedSciSportSExerc.2006,38(2):250-5

[8]Yamanos,EtoD,MukaiK,etal.Effect of high intensxty training on a aerobic capacity Of middle qlutea muscle in Thoroughbred horses.ResVetSei.2004:76(2):139-44

[9]W.X. Peng. Analysis On Molecular Characteristics Of Wood Extractives From Eucalyptus Urophydis Biomass. BioTechnology : An Indian Journal, 2013, 7(12), 559-564.

[10]L.S. Wang, W.X. Peng, M.L. Zhang and Z. Lin. Separation Characteristics of Lignin from Eucalyptus Lignincellulose for Medicinal Biocellulose Preparation. Journal of Pure and Applied Microbiology, 2013, 7 (SI), 59-66. 